\title{
Acoustic Biosensor for Discrimination of Pathogens according to Gram-Principle ${ }^{\dagger}$
}

\author{
Vladislav Lemozerskii *, Tatiana Zimina and Alena Gagarina \\ St. Petersburg Electrotechnical University “LETI”, St Petersburg 197022, Russia; \\ * Correspondence: lme812@gmail.com; Tel.: +7-981-971-4863 \\ + Presented at the 1st International Electronic Conference on Biosensors, 2-17 November 2020; Available \\ online: https://iecb2020.sciforum.net/.
}

Received: date; Accepted: date; Published: date

\begin{abstract}
The microacoustic methods of biomedical analysis, implemented on piezoelectric crystals and ceramics are becoming increasingly perspective due to potential of integration into laboratorieson-a-chip, biochips and biosensors, as functional elements of biosensors. An important stage in diagnostics of infectious diseases is the identification of pathogens. One of possible applications of such sensor is an alternative to time and labor consuming Gram method of discriminating bacteria according to composition of their cell walls. Thus, bacteria which in a procedure of Gram staining, do not decolored after application of dye solution, are classified as Gram-positive $(G(+))$. They are surrounded with the thick peptidoglycan layer, pulpy and dampening acoustic waves. While Gramnegative $(G(-))$ bacteria, which acquire red color in Gram procedure, are covered with thin and springy layer, demonstrating resonance effects when interact with acoustic fields. Thus, $G(+)$ and $\mathrm{G}(-)$, which differently colored in Gram procedure are also react differently to external acoustic field: for $\mathrm{G}(-)$ bacteria, this is a sharp decrease in the Q-factor of the "resonator-suspension" system and a shift of the resonance curve to lower frequencies. While for $G(+)$ bacteria, although a certain shift of the resonance curve was also observed, but the bandwidth of resonance curve practically did not change. This effect was studied for L. acidophilus $(\mathrm{G}(+))$ and E. coli $(\mathrm{G}(-))$ bacilli with quarts resonators of $4 \mathrm{MHz}, 5 \mathrm{MHz}$ and $10 \mathrm{MHz}$. The biosensor was tested using Lactobacillus fermentum, E. coli M-17, Bifidobacterium bifidum, Burkholderia cepacian and Staphylococcus aureus. At this stage it has been demonstrated that the method is particularly effective for discriminating bacteria of the similar shape, such as, for example cocci. The discrimination of Gram factor for cocci and bacilli was less accurate and needs further studies for selection of precise resonance frequencies.
\end{abstract}

Keywords: acoustic analysis; quarts resonators; Gram positive bacteria; Gram negative bacteria; identification of microorganisms

\section{Introduction}

The microacoustic methods of biomedical analysis, implemented on piezoelectric crystals and ceramics are becoming increasingly perspective due to potential of integration, as functional sensor elements, into the hybrid analytical devices and laboratories-on-a-chip [1-3]. One of possible applications of such sensor is alternative to identification of bacteria according to Gram method, which enables to discriminate them according to composition of their cell walls [4]. Thus, bacteria which in a procedure of Gram staining, do not decolored after bleeding of dye at a discoloration stage, are classified as Gram-positive $(\mathrm{G}(+))$ [5]. The protection of the color is due to thick $(20-30 \mathrm{~nm})$ layer of peptidoglycan with a fibrous structure in the bacterial cell wall which retains the coloring agent (Figure 1). The signature feature of $\mathrm{G}(+)$ bacteria is the presence of teichoic and lipoteichoic 
acids. However, despite a thicker layer of peptidoglycan, $G(+)$ bacteria are more susceptible to antibiotics.

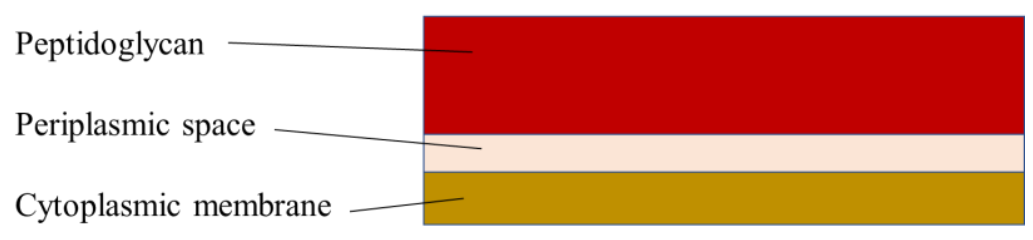

Cytoplasm

Figure 1. Shell structure of gram-positive bacteria.

Most of the human pathogenic microorganisms are $G(+)$. Thus, six genera of $G(+)$ microorganisms are typical human pathogens, two of them are cocci (streptococci and staphylococci), the others are bacilli-a rod-like bacteria. The typical members of $\mathrm{G}(+)$ bacilli are: Bacillus subtilis, Bacillus cereus, Bacillus anthracis, etc.

$\mathrm{G}(-)$ bacteria are a group of bacteria decolored when stained with crystal violet in the Gram's method [6]. They are characterized with cell walls consisting of a thin layer of peptidoglycan, clamped between the inner membrane of cytoplasmic cells and the outer bacterial membrane (Figure 2). The signature feature of Gram-negative bacteria is the absence of teichoic and lipoteichoic acids, accordingly $\mathrm{G}(-)$ bacteria can't retain the purple dye after decoloration stage. The alcohol used in this stage degenerates the outer membrane of $\mathrm{G}(-)$ cells and makes the cell wall more porous and unable to hold crystal violet. The peptidoglycan layer of $G(-)$ bacteria is thinner than that in $G(+)$ and is clamped between the inner and outer membranes, causing them to insert a contrasting agent (safranin or fuxin) and look red or pink.

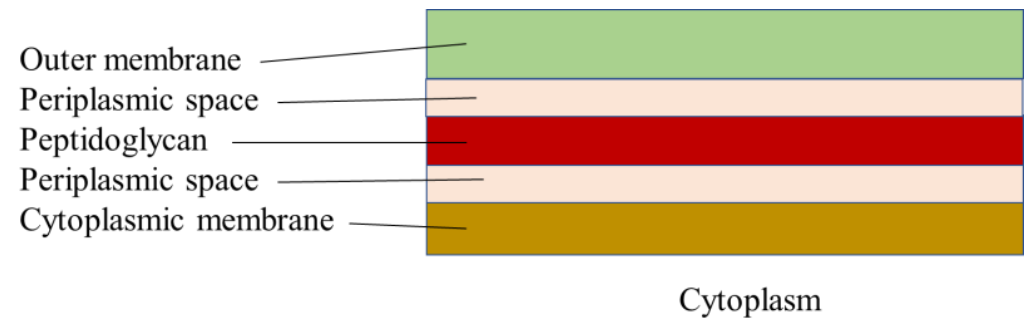

Figure 2. Shell structure of gram-negative bacteria.

The typical members of $G(-)$ bacteria are the proteobacteria (Escherichia coli (E. coli), Salmonella, Shigella, Pseudomonas, Moraxella, Helicobacter, Stenotrophomonas, Bdellovibrio, Legionella), cyanobacteria and some types of bacilli (Hemophilus influenzae, Klebsiella pneumoniae, Legionella pneumophila, Pseudomonas aeruginos), etc.

The Gram staining is not an absolutely accurate tool to identify bacteria. This method is used for preliminary diagnostics or to find out number of bacteria in the clinical sample. It can't identify bacteria at the species level and should not be used as the only way to differentiate bacteria for most diseases. The main disadvantage of the Gram staining is unsuitability of samples for research after the procedure. In addition, the method is labor intensive. However, it seems useful as additional indicator in identification of microorganisms, for example in implementing a new generation hybrid device for microbiological analysis [2,3]. This is due to a strong discriminative feature of these two groups of bacteria, i.e., the rigidly of the cell wall, which is a subject of physical methods of measurement.

Therefore, there is a need for an alternative and less time-consuming miniature method of differentiating bacteria by Gram, or by rigidity of their cell wall. The acoustic resonance method is a promising approach as an alternative to classical Gram method, which surpasses it by the speed, and 
integrability. But there are limitations which connected with variations of shapes of bacteria, which could contribute into the result [7].

Thus, in this paper the method of bacteria discrimination based on their unequal response to different external mechanical influences and which is by the result would be a double to the Gram staining method is discussed.

\section{Theoretical Background}

An implementation of microacoustic sensor for bacteria needs a selection of element for generation of appropriate acoustic fields. A quartz resonator is an electronic device in which the piezoelectric effect and the phenomenon of mechanical resonance are used to construct a high-quality resonant element of an electronic circuit $[8,9]$.

On a plate, a thin cylinder, a ring or a bar cut from a quartz crystal with a certain orientation relative to the crystallographic axes of a single crystal, two or more electrodes are depositedconductive metal strips made by sputtering in vacuum or by burning a metal film on a given crystal surface [10].

The resonator is mechanically mounted in the nodes of the working mode of vibration in order to reduce the loss of vibrational energy through the crystal mount. For other modes of vibration, the nodes of natural vibrations are located in other places of the crystal, and therefore other vibration modes are suppressed. For the working mode of oscillations, the crystal has a certain natural resonant frequency of mechanical vibrations, and at this frequency, the $Q$-factor of the mechanical resonator is very high [11].

When voltage is applied to the electrodes, bending, compression, or shearing occurs due to the piezoelectric effect, depending on how the crystal is cut relative to the crystallographic axes, the configuration of the exciting electrodes, and the location of the attachment points (Figure 3) [12].

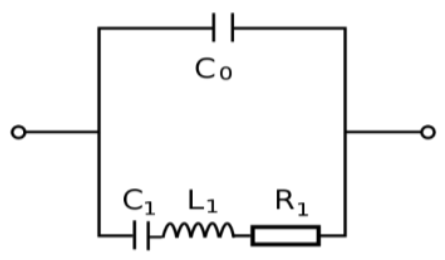

Figure 3. An equivalent circuit of a quartz resonator: $\mathrm{C}_{0}-$ intrinsic capacitance of a crystal; $\mathrm{C}_{1}$ and $\mathrm{L}_{1}$ are the equivalent capacitance and inductance of the mechanical oscillatory system of the resonator; $\mathrm{R}_{1}$ - equivalent loss resistance.

The natural oscillations of the crystal as a result of the inverse piezoelectric effect induce an additional electromotive force, emf, on the electrodes and therefore the quartz resonator behaves electrically like a resonant circuit-an oscillatory circuit composed of capacitors, inductance and resistor, and the quality factor ( $Q$-factor) of this equivalent electric circuit is very large and close to the $Q$-factor of its own mechanical crystal vibrations [11].

If the frequency of the applied voltage is equal to or close to the frequency of the intrinsic mechanical vibrations of the plate, the energy consumption for maintaining the plate vibrations is much lower than that with a large difference in frequency. This also corresponds to the behavior of the electric oscillatory circuit [9].

The advantages of quartz-based resonators include a low loss tangent and very low mechanical losses (i.e., high mechanical Q-factor). In the best samples of a quartz crystal, this can be $10^{6}-10^{7}$. This feature provides high frequency selectivity of quartz resonators. Due to the fact that the reserves of natural quartz are limited, it is grown artificially by the hydrothermal method [12]. Crystallization is carried out in high-capacity steel autoclaves from aqueous alkaline solutions at a temperature of 350$400{ }^{\circ} \mathrm{C}$ and pressures of the order of $10^{8} \mathrm{~Pa}$. Due to the low solubility of silica in aqueous solutions, one growing cycle can last several months [13].

\section{Materials}


Suspensions of $\mathrm{G}(+)$ and $\mathrm{G}(-)$ bacteria were prepared by diluting a dry bacteria sample ( 5 doses or other) in $1 \mathrm{~mL}$ of physiological solution, obtaining thus a stock solution for further dilution.

The following microorganisms were used in experiments:

Lactobacillus fermentum [14], a microbial mass of a live, antagonistically active strain of lactobacilli, lyophilized in a culture medium with the addition of a protective sucrose-gelatin-milk drying environment. 1 doze contains not less than $2 \times 10^{9}$ living bacteria, protection medium contains (per $1 \mathrm{~L}$ of the medium): gelatin up to $8.5 \%$ vol; sugar up to $28 \%$ weight; skimmed milk powder up to 14\% weight; code EAN: 4602784000076; No LS-002098, 2011-10-25; Manufacturer: «Scientific industrial Consortium «Microgen», 115088, Moscow, 1-st. Dubrovskaya str., house 15, Russia.

E. coli M-17 [15], from Colibacterin siccum, 1 doze contains not less than $10 \times 10^{9}$ living bacteria. Protection substances in the prepartion: gelatin, sugar. Manufacturer: «Scientific industrial Consortium «Microgen», 115088, Moscow, 1-st. Dubrovskaya str., house 15, Russia.

Bifidobacterium bifidum [16], from Bifidum bacterin, 1 doze contains not less than $10^{7}$ living bacteria and protection components: gelatin, sugar, dry milk. Manufacturer: code EAN: 4607006820289, No P N000063/01, 2011-02-21, Vitapharma Ltd., Russia.

Burkholderia cepacia, the strain prepared at St. Petersburg Pasteur Institute. Growth Medium: Agar/Meet-peptone broth.

Staphylococcus aureus, the strain prepared at St. Petersburg Pasteur Institute. Growth Medium: Agar/Meet-peptone broth

\section{Experimental}

The experimental setup shown in Figure 4 comprises: an electric oscillation generator (PV6503), an acoustic sensor based on a quartz resonator, a $1.5 \mathrm{M} \Omega$ resistor connected in series to the resonator.

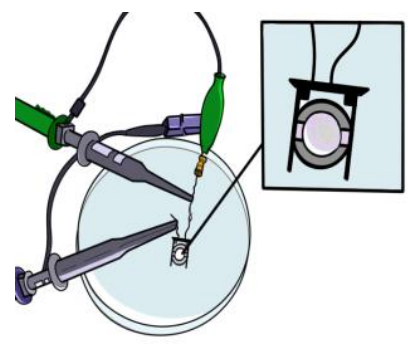

(a) (b)

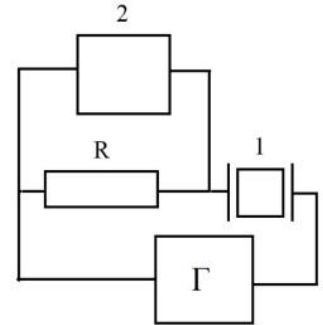

(c)

Figure 4. The experimental setup for acoustic studies of suspensions of microorganisms: (a)arrangement of electrodes; (b)-view of quartz resonator; (c)-block scheme of the acoustic measurement setup. 1 -acoustic sensor, 2-sample, $R$-resistor, $\Gamma$-generator PV6503.

Suspensions of $G(+)$ and $G(-)$ bacteria of different volumes were loaded to the test system in order to determine the optimal load volume. The suspensions of bacteria were prepared by diluting a stock solution as described above, using an automatic pipette and physiological saline solution. The loading of suspensions of defined volume was made using a precision low volume automatic pipette. The range of volumes was $5 \mu \mathrm{L}, 10 \mu \mathrm{L}, 15 \mu \mathrm{L}$.

The procedure of samples loading onto the sensor surface was strictly controlled by volume. The volumes were applied on a quartz plate, after which the amplitude-frequency dependence recorded through a frequency meter was recorded on resistor included in the circuit.

The characteristics of measured samples according to Gram classification are presented in Table 1. 
Table 1. Nomenclature of used samples.

\begin{tabular}{clcc}
\hline No. & Name of Species/Strain & The Form & Gram Classification \\
\hline 1 & Lactobacillus fermentum & Rod-shaped & $\mathrm{G}(+)$ \\
2 & Escherichia coli M-17 & Rod-shaped & $\mathrm{G}(-)$ \\
3 & Bifidobacterium bifidum & Rod-shaped & $\mathrm{G}(+)$ \\
4 & Burkholderia cepacia & Rod-shaped & $\mathrm{G}(-)$ \\
5 & Staphylococcus aureus & Spherical & $\mathrm{G}(+)$ \\
\hline
\end{tabular}

\section{Results and Discussion}

Before start of the experiments, the quartz resonator was calibrated with a nominal resonant frequency of $4 \mathrm{MHz}$ and the amplitude-frequency spectra (ASF) were recorded for the decoupled resonator. It was found that its resonant frequency is $3.9989 \mathrm{MHz}$.

Then, ASF measurements were made for the resonator loaded with water and alcohol (Figure 3) in order to check an influence of the sample viscosity, which differs for water and alcohol. The results are presented for reduced values, $k=f / f f_{e s}$, where $f$-is the measured frequency, fres is the resonance frequency of the quarts resonator.

Similar experiments were carried out with suspensions of microorganisms. Figure 5 shows that with an increase of the suspension volume as applied to $G(+)$ Lactobacillus fermentum, the position of the resonance peak is slightly shifted to the region of lower frequencies relative to the value of the resonance frequency for the empty resonator.

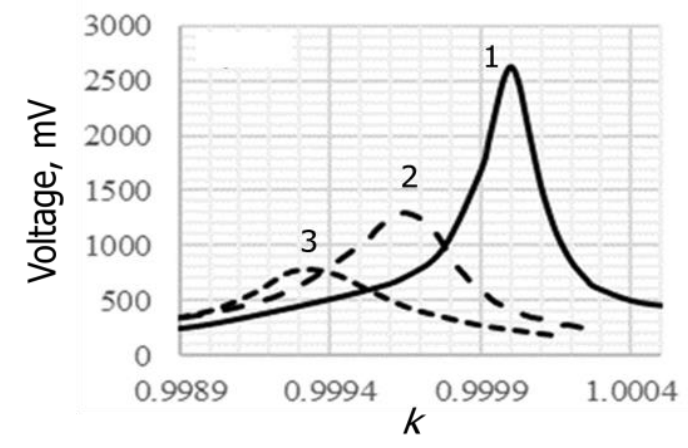

Figure 5. Amplitude-frequency spectra (AFS), for an empty resonator, water (5 $\mu \mathrm{L})$ and alcohol (5 $\mu \mathrm{L}) ; 1$-empty resonator, 2 -alcohol, 3 -water; $k$-reduced spectra, $f / f_{\text {res. }}$.

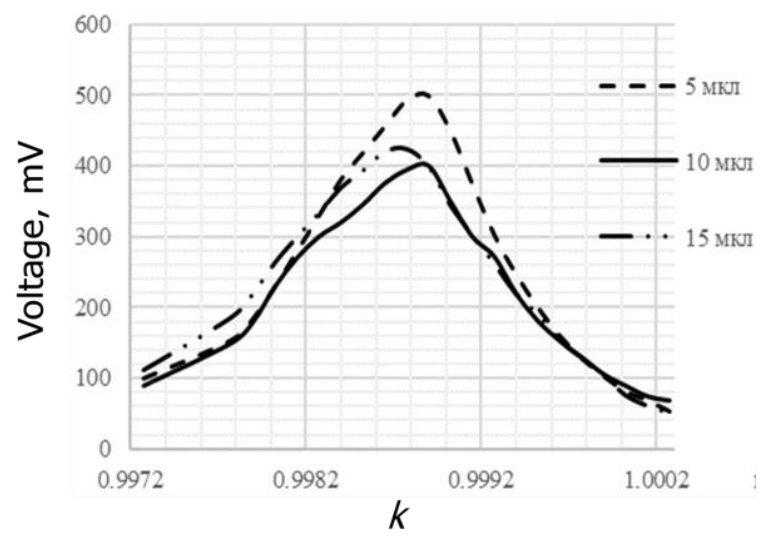

Figure 6. Frequency response for the load of various volumes of $\mathrm{G}(+)$ bacteria Lactobacillus fermentum.

Figure 7 shows that for $G(+)$ bacteria, a slightly shifted peak maximum corresponding to the resonant frequency is characteristic. 


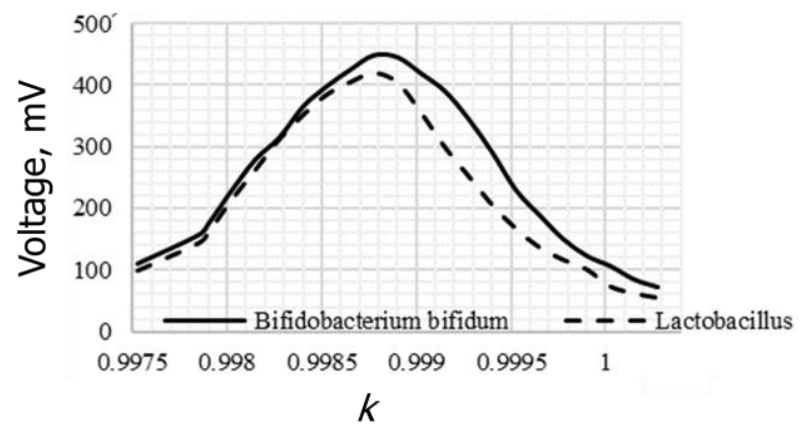

Figure 7. Frequency response for gram-positive bacteria Bifidobacterium bifidum and Lactobacillus fermentum.

Testing of $G(+)$ and $G(-)$ bacilli showed that the resonance frequency peak position for $G(-) E$. coli is considerably shifted to the region of lower frequencies, relative to both gram-positive bacteria, Bifidobacterium bifidum and Lactobacillus fermentum, and the empty resonator (Figure 8).

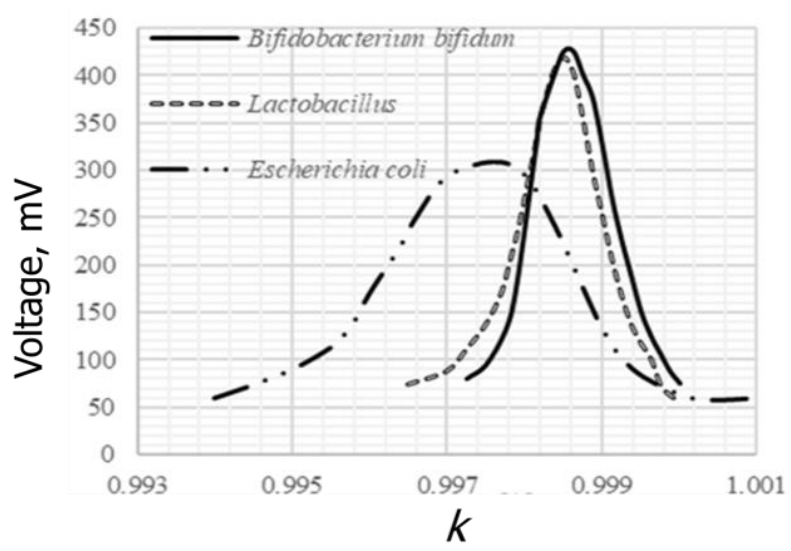

Figure 8. Experimental dependences of the amplitude on the reduced frequency for suspensions of $\mathrm{G}(+)$ (Bifidobacterium bifidum and Lactobacillus fermentum) and $\mathrm{G}(-)$ (Escherichia coli) bacteria loaded on resonator in $5 \mu \mathrm{L}$ volume.

Moreover, $\mathrm{G}(-)$ bacterium is characterized by a significant increase in the bandwidth of AFC, which indicates a sharp decrease in the $Q$-factor of the system (Figure 9).

The observed relations demonstrate that suspensions of bacteria add such an element to the resonance system, in which energy dissipation occurs during the propagation of acoustic waves. The observed energy dissipation during the propagation of acoustic waves in a thin quartz disk cannot be explained solely by the different viscosities of the studied suspensions, since according to Einstein's law [17], the viscosity of a suspension of smooth spheres depends on the volume concentration of solids and does not depend on the particle size. It could be concluded that differences in the values of the amplitudes of the reduced frequency for $G(+)$ and $G(+)$ bacteria are mostly associated with the peculiarities of the chemical structure of bacterial membranes and their rigidity.

The spherical $G(+)$ and rod-shaped $G(-)$ bacteria, were tested to compare the shifts of resonance frequency. Figure 7 shows that for this type of bacteria the shift to lower frequencies for both types of bacteria remains, but there is no significant difference between the value of this shift and the bandwith for Staphylococcus aureus, G(+) and Burkholderia cepacian, G(-). This may be directly related to the differenceies in the rigidity of their cell walls and the shape. Staphylococcus aureus belongs to spherical bacteria (cocci), and Burkholderia cepacia to rod-shaped (bacilli) bacteria. The simultaneous effect of both factors decreased the selectivity of method based on the effect of resonance frequency shift. 


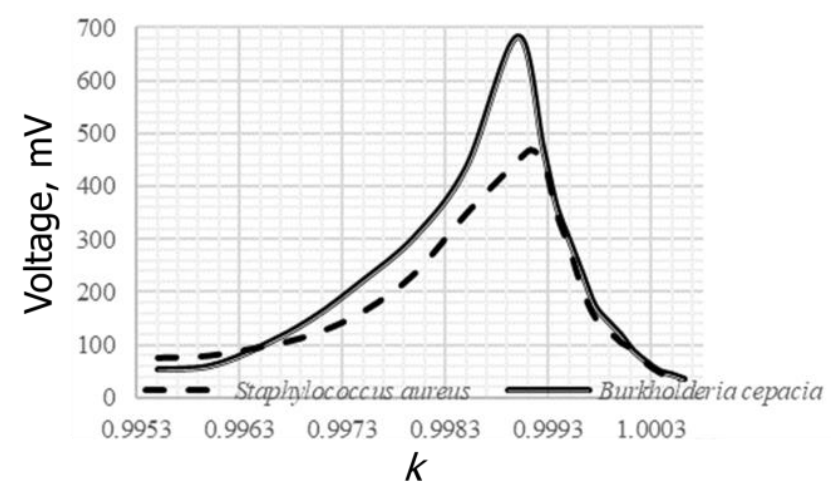

Figure 9. Experimental dependences of the amplitude on the reduced frequency for suspensions of $\mathrm{G}(+)$ (Staphylococcus aureus) and $\mathrm{G}(-)$ (Burkholderia cepacia) bacteria loaded as a volume of $5 \mu \mathrm{L}\left(10^{9}\right.$ CFU).

\section{Conclusions}

The study has shown that, due to the different structure of cell walls, gram-positive and gramnegative bacteria react differently to external disturbance: for $\mathrm{G}(-)$ bacteria, a sharp decrease in the quality factor of the resonator-suspension system and a shift of the resonance curve to lower frequencies were observed; for gram-positive bacteria, a shift in the resonance curve was also observed, but to a lower extent than for $\mathrm{G}(-)$ bacteria, and the bandwidth was practically unchanged.

However, these trends were observed for bacteria of the same shape - the bacteria studied in the first part of the experiment (Bifidobacterium bifidum, Lactobacillus fermentum, and Escherichia coli) were rod-shaped bacilli. When comparing bacteria of different shapes: spherical Staphylococcus aureus and rod-shaped Burkholderia cepacia, characteristic shifts of the resonance curves were also observed, but no significant changes in the bandwidth were noted. These results were obtained for the resonator with resonance frequency of $4 \mathrm{MHz}$.

The experimental results obtained, demonstrated that the acoustic resonance method is suitable for differentiation of bacteria, being discriminated by Gram, particularly this is valid for bacteria of the similar shape. These results were confirmed for quarts resonator with the resonance frequency of $4 \mathrm{MHz}$. To further increase the selectivity of the method, it is important to test the wide range of resonators with the resonant frequencies of the range at least from $1 \mathrm{MHz}$ to $100 \mathrm{MHz}$, which would enable to select the conditions most sensitive to the cell wall rigidity, than for the shape of the total cell. This will create a background for development of integrable elements for laboratories-on-a-chip and miniature diagnostic devices [18].

Author Contributions: Conceptualisation, V.L., T.Z.; methodology, V.L., T.Z., A.G.; investigation, A.G., V.L.; writing-original draft preparation, V.L., A.G.; writing-review and editing, T.Z. All authors have read and agreed to the published version of the manuscript.

Acknowledgments: The authors acknowledge with thanks colleagues from St. Petersburg Pasteur Institute, L. A. Kraeva and G. N. Hamdulaeva for their help in carrying experiments with Staphiloccus aureus and Burkholderia cepacia.

Conflicts of Interest: The authors declare no conflict of interest.

\section{References}

1. Adem, O.; Rufo, J.; Guo, F.; Gu, Y.; Li, P.; Lata, J.; Huang, T.J. Acoustic tweezers for the life sciences. Nat. Methods 2016, 15, 1021-1028.

2. Zimina, T.M.; Luchinin, V.V. Microsystems for express analysis. J. Anal. Chem. 2011, 66, 1136-1158, doi:10.1134/S1061934811100236.

3. Zimina, T.M.; Luchinin, V.V.; Sitkov, N.O.; Gvozdev, Y.A.; Lemoserskii, V.E.; Orekhov, Y.D. Hybridintegrated lab-on-a-chip for total express-microbiological analysis: State of the art and the nearest perspectives. In Proceedings of the 2019 IEEE Conference of Russian Young Researchers in Electrical and 
Electronic Engineering (EIConRus), St. Petersburg and Moscow, Russia, 28-30 January 2019; Volume 2140, doi:10.1063/1.5122009.

4. Panawala, I. Difference between Gram Positive and Gram Negative Bacteria. 2017. Available online: https://www.researchgate.net/publication/315757324 (accessed on 15 April 2019).

5. Gram-Positive Bacteria. [Electronic Resource]/Access mode. Available online: https://en.wikipedia.org/wiki/Gram-positive_bacteria. free.-Zagl. from the screen (accessed on).

6. Gram-Negative Bacteria. [Electronic Resource]/Access mode. Available online: https://en.wikipedia.org/wiki/Gram-negative_bacteria. free.-Zagl. from the screen (accessed on).

7. Semenov, A.I.; Soloviev, A.V.; Zimina, T.M. Investigation of the possibility of identifying bacteria using an acoustic sensor based on a quartz resonator. Biotechnosphere 2017, 48, 29-33.

8. Yarlagadda, P.; Yang, S.-F.; Leequarts, K.M. The Resonant Properties of AT-Cut Quartz Resonator and its Application. Appl. Mech. Mater. 2016, 411-414, 1631-1634.

9. Laschitch, A.; Johannsmann, D. High frequency tribological investigations on quartz resonator surfaces. J. Appl. Phys. 1998, 85, 3759-3765.

10. Schweber, B. Quartz Crystals and Oscillators. Part 1: Crystal Basics. 2018. Available online: https://www.analogictips.com/quartz-crystals-oscillators-part-1-crystal-basics-faq (accessed on).

11. Reference Data for Radio Engineers, 9th ed.; Elsevier: Amsterdam, The Netherlands, 2002; Chapter 1. ISBN 978-0-7506-7291-7.

12. Bottom, V.E. Introduction to Quartz Crystal Unit Design; Van Nostrand Reinhold: New York, NY, USA, 1989.

13. Sunagawa, I.; Iwasaki, H.; Iwasaki, F. Growth and Morphology of Quartz Crystals: Natural and Synthetic; Terrapub: Tokyo, Japan, 2009.

14. Pan, D.D.; Dao, D.Z.; Zeng, X.Q.; Yan, Y.T. Characterisation of Lactobacillus fermentum SM-7 isolated from koumiss, a potential probiotic bacterium with cholesterol-lowering effects. J. Sci. Food Agric. 2011, 91, 512518.

15. Sagitova, A.; Yaminsky, I.; Meshkov, G. View of the bacterial strains of Escherichia coli M-17 and its interaction with the nanoparticles of zinc oxide by means of atomic force microscopy. J. Phys. Conf. Ser. 2016, 741, 012059.

16. Seockmo, K.; Myeong, S.P.; Geun, E.J.; Hyun, Y.J. Review on Bifidobacterium bifidum BGN4: Functionality and Nutraceutical Applications as a Probiotic Microorganism. Int. J. Mol. Sci. 2016, 17, 1544.

17. Zimina, T.M.; Smith, R.M.; Myers, P.; King, B.W. Effects of kinematic viscosity of the slurry on the packing efficiency of peek microbore columns for liquid chromatography. Chromatographia 1995, 40, 662-668.

18. Lemozerskii, V.E.; Zimina, T.M.; Sitkov, N.O.; Koigerov, A.S.; Pudova, A.V. Sensor/Actuator Elements Based on SAW Principle for Flexible Disposable Laboratories-on-a-Chip for Biomedical Analysis. In Proceedings of the 2019 IEEE Conference of Russian Young Researchers in Electrical and Electronic Engineering (EIConRus), St. Petersburg and Moscow, Russia, 28-30 January 2019; pp. 1306-1309, doi:10.1109/EIConRus.2019.8656999.

Publisher's Note: MDPI stays neutral with regard to jurisdictional claims in published maps and institutional affiliations.

(C) 2020 by the authors. Submitted for possible open access publication under the terms and conditions of the Creative Commons Attribution (CC BY) license (http://creativecommons.org/licenses/by/4.0/). 